UROLOGICAL CANCER

\section{Atezolizumab effective against advanced disease}

Patients with metastatic urothelial carcinoma have few established treatment options, and standard-of-care therapy typically prolongs survival by only $9-15$ months. Now, findings of a phase II clinical trial indicate that a subset of these patients respond to the anti-programmed cell-death 1 ligand-1 (PD-L1) humanized monoclonal antibody atezolizumab. In this single-arm trial, 315 patients with inoperable, advanced-stage or metastatic urothelial carcinoma, whose disease had progressed despite receiving previous platinum-based chemotherapy, were enrolled. The extent of pretreatment PD-L1 expression upon enrolment was determined using immunohistochemical analyses of tumour-infiltrating lymphocytes (TILs) in biopsy samples. Patients received 3-weekly doses of atezolizumab: $26 \%$ of patients with $>5 \%$ PD-L1-positive TILs and $18 \%$ of those with $>1 \%$ PD-L1-positive TILs had an objective response to treatment. Although no comparator arm was included, this value is substantially higher than the $\sim 10 \%$ of patients who respond to frequently used cytotoxic agents, as demonstrated by previous research. At the time of publication, patients had a median response duration of 11.7 months, and $84 \%$ of patients had an ongoing response. Grade 3 or 4 treatment-related adverse events ocurred in $16 \%$ of patients, with grade 3 or 4 immune-related adverse events, including pneumonitis, rash and dyspnoea in $5 \%$.

These findings indicate that atezolizumab provides a viable alternative to cytotoxic chemotherapies for patients with metastatic urothelial carcinoma, despite substantial pretreatment with other therapies, and is particularly effective in patients with elevated PD-L1 expression on TILs. Longer-term follow up data on the outcomes of patients who continue to respond to atezolizumab are eagerly awaited.

Peter Sidaway

This article is modified from the original in Nat. Rev. Urol. (http://dx.doi.org/10.1038/nrurol.2016.60).

ORIGINAL ARTICLE Rosenberg, J. E.et al. Atezolizumab in patients with locally advanced and metastatic urothelial carcinoma who have progressed following treatment with platinum-based chemotherapy: a single-arm, multicentre, phase 2 trial. Lancet http://dx.doi.org/10.1016/S01406736(16)00561-4 (2016). 